\title{
Design and Implementation of An Improved Camera Mounted Remote Controlled Quadcopter
}

\author{
Ganiyu Adedayo Ajenikoko ${ }^{1}$, Victor Uchenna Nwagbara ${ }^{2}$, Ezekiel Olusegun Ojekunle ${ }^{3}$, Babajide Akinjobi ${ }^{4}$, \\ Oladimeji S. Adepoju ${ }^{5}$, Peter Ojekunle ${ }^{6}$, Oluwagbenga Samson Omotosho ${ }^{7}$, Olaide A. Osinubi ${ }^{8}$ \\ 1.2,3,4,5,6,7,8 Department of Electronic and Electrical Engineering, Ladoke Akintola University of Technology, \\ P.M.B, 4000, Ogbomoso, Nigeria.
}

Corresponding Email: ajeedollar@gmail.com

\begin{abstract}
Aeronautics and other studies on the science of aircraft have advanced to the point where Unmanned Aerial Vehicles (UAVs) or drones are now being extensively designed. However, such aircrafts are large and not maneuverable in tight spots especially fixed wing aircraft, therefore, the design of multi-rotors are now being considered. It is inherent that small unmanned aircraft with optimal maneuverability and the ability to carry small payloads such as cameras should be designed for operations in places where a full-size aircraft are either too big or too expensive to be deployed. One of the types of Small Unmanned Aerial Vehicle (SUAV) is a Quadcopter, which can be implemented in different applications. Quadcopters are rapidly gaining interest due to their stability, low cost in building, handling capabilities and agility. Uses of such craft include aerial photography or surveillance, ground surveillance and mapping, package delivery, for rescue operations and as a research tool into ways of designing more advanced aircraft. This study therefore designed and implemented an improved industry-grade Small Unmanned Aerial Vehicle (SUAV) multi-rotor Quadcopter. Quadcopter structure model, basic components, hovering stability, dimensions, and description of basic principles of quadcopter were discussed. The study showed that SUAVs are useful across a broad range of applications.
\end{abstract}

Keywords: Unmanned Aerial Vehicles, Aeronautics, Aircraft, Multi-rotors, Quadcopter, Surveillance, Aerial Photography, Small Unmanned Aerial Vehicle.

DOI: $10.7176 / \mathrm{CEIS} / 11-2-08$

Publication date: April $30^{\text {th }} 2020$

\section{INTRODUCTION}

An Unmanned Aerial Vehicle (UAV), also known as Unmanned Aircraft System (UAS) or Drone is an aircraft without a human pilot on board. A UAV's flight may be controlled remotely by an operator located on the ground or in another vehicle by onboard computers [1]. Unmanned Aerial Vehicles are often preferred for missions that are too dull, dirty or dangerous for manned aircraft. They are mostly found in military and special operation applications, though UAVs are increasingly finding uses in civil and recreational applications, such as policing and surveillance, aerial photography, and drone racing $[12,13]$.

UAVs range from small handheld aircraft with an altitudinal range of up to $600 \mathrm{~m}$ to large orbital low earth orbit craft. The class of UAVs include fixed wing, single and multirotor craft. A multirotor or multi-copter is a helicopter with multiple rotors and propellers providing uplift, thrust and maneuverability [3]. Multi-rotors are classified as rotorcraft, as opposed to fixed-wing aircraft, because their lift is generated by a set of rotors (vertically oriented propellers). Multi-rotors differ from conventional helicopters which use rotors that are able to vary the pitch of their blades dynamically as they move around the rotor hub. They use independent variation of the speed of each rotor to achieve control $[1,4,7,11]$.

This class of UAVs is commonly referred to as miniature or Small Unmanned Aerial Vehicle (SUAV) as they range from Micro Air Vehicles (MAVs) that can be carried by one person to man-portable UAVs that can be carried and launched like an infantry man-portable air-defense system. One of the SUAV that can perform better in air defense system is the multi-rotor Quadcopter [1, 2, 5].

A quadcopter also called a quadrotor helicopter or quadrotor is a multirotor helicopter lifted and propelled by four rotors. The motors are mounted in the same plane, on the helicopter, forming a square [6, 10]]. This allows the quadcopter to lift vertically without rotating on itself. There are several advantages of quadcopters over comparably-scaled helicopters. The fact that four motors are involved reduces the minimum size of the propellers on each motor. This means that in case the quadcopter hits anything, the damage is significantly lesser than if a similar sized helicopter crashed. Moreover, the present day uses of quadcopters are 
diverse as these crafts are being used for surveillance and reconnaissance, amateur model aircraft projects, aerial photography, flight control theory, navigation, real time systems, robotics and aerodynamic thrust $[5,8,9]$.

\section{QUADCOPTERS}

A quadcopter, as shown in Figure 1, also called a quadrotor helicopter or quadrotor, is a multirotor helicopter that is lifted and propelled by four rotors. Quadcopters are symmetrical and embody the simplest principle of operation for controlling roll, pitch, yaw and motion. Quadcopters generally use two pairs of identical fixed pitched propellers; two clockwise (CW) and two counter-clockwise (CCW) [3]. These use independent variation of the speed of each rotor to achieve control. By changing the speed of each rotor, it is possible to specifically generate a desired total thrust; to locate for the center of thrust both laterally and longitudinally; and to create a desired total torque, or turning force $[2,7,9]$.

In the late $2000 \mathrm{~s}$, advances in electronics allowed the production of cheap lightweight flight controllers, accelerometers and gyroscopes, global positioning system and cameras. This resulted in a rapid proliferation of small, cheap consumer quadcopters along with other multi rotor designs [3]. Quadcopter designs became popular in UAVs or drone research. With their small size and maneuverability, these quadcopters can be flown indoors as well as outdoors. At a small size, quadcopters are cheaper and more durable than conventional helicopters due to their mechanical simplicity. Their smaller blades are also advantageous because they possess less kinetic energy, reducing their ability to cause damage $[4,8,10]$.

For small-scale quadcopters, this makes the vehicles safer for close interaction. It is also possible to fit quadcopters with guards that enclose the rotors, further reducing the potential for damage. However, as size increases, fixed propeller quadcopters develop disadvantages over conventional helicopters. Increasing blade size increases their momentum. This means that changes in blade speed take longer, which negatively impacts control [3]. At the same time, increasing blade size improves efficiency as it takes less energy to generate thrust by moving a large mass of air at a slow speed than by moving a small mass of air at high speed. Therefore, increasing efficiency comes at the cost of control. Helicopters do not experience this problem as increasing the size of the rotor disk does not significantly impact the ability to control blade pitch $[1,9,11]$.

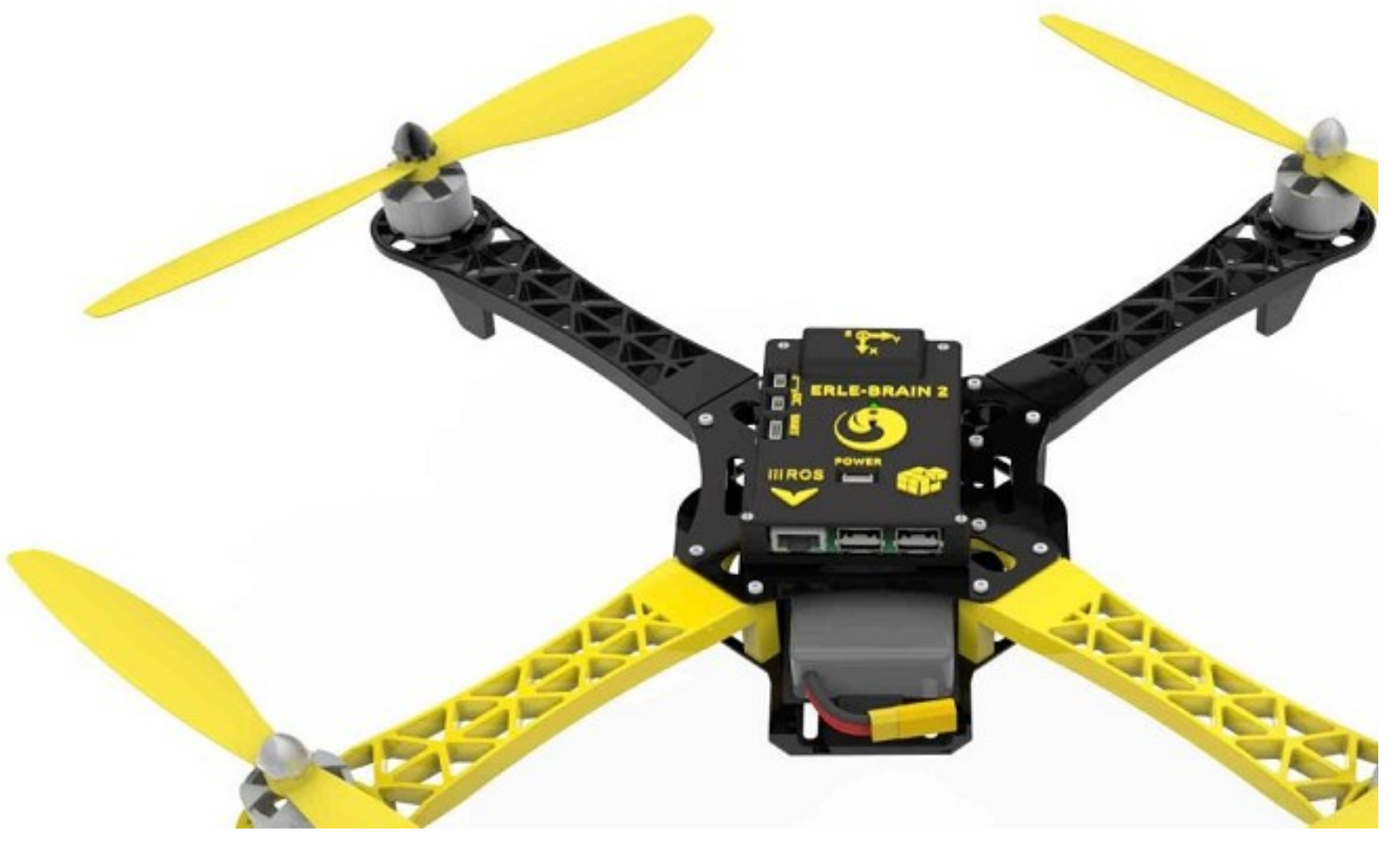

Figure 1: A Quadcopter

Quadcopters can be classified by wheelbase size; from $4 \mathrm{~mm}$ to over $1 \mathrm{~m}$, frame configuration; ' $\mathrm{X}$ ', '十' or ' $\mathrm{H}$ ' frame, payload capacity; from $0.4 \mathrm{~kg}$ to almost $60 \mathrm{~kg}$ and function; aerial photography, First Person View (FPV), drone racing and acrobatics, surveillance and reconnaissance. In addition, a quadcopter is made up of basically three systems $[8, .10]$ :

i. The Power Conversion System: This consists of the battery, motors, speed controllers and the propellers 
ii. The Mechanical System: This system consists of everything in the quadcopter, because every part of the quadcopter has mechanical properties and

iii. The Control System: This system consists of the transmitter/ receiver combinations (both movement and video), flight controller and various sensors.

\section{a. Principles of Operation}

Quadcopters use two sets of identical pitched propellers-2 clockwise and 2 counterclockwise. These produce both a thrust and torque at its center of rotation, as well as a drag force opposite to the vehicle's direction of flight. Control of vehicle motion is achieved by altering the rotation rate of one or more rotor discs, thereby changing its torque load and thrust/lift characteristics [4]. If all rotors are spinning at the same angular velocity for a vertical lift, with rotors 1 and 3 rotating clockwise and rotors 2 and 4 counterclockwise, the net aerodynamic torque, and hence the angular acceleration about the yaw axis, is exactly zero, indicating that there is no need for a tail rotor- like conventional helicopters. Figure 2 shows the schematic diagram of each motor's reaction torque $[1,9]$.

In addition, four best controllable variables can be chosen and related to the four basic movements which allow the quadcopter to reach a certain height and attitude. These controllable variables are described as follows $[2,5,12,13]$ :

i. Throttle: This increases or decreases all the propeller speeds by the same amount. It leads to a vertical force which raises or lowers the quadcopter. If the quadcopter is in horizontal position, the vertical direction of the inertial frame coincides. Otherwise the provided thrust generates both vertical and horizontal accelerations in the inertial frame

ii. Yaw: This is controlled by turning up the speed of the regular rotating motors and taking away power from the counter rotation; taking away the same amount of power put on the regular rotors produces no extra lift, but since the counter torque is now less, control becomes a matter of which motor gets more power and which one gets less. The yaw of a quadcopter is controlled on the transmitter using the rudder controls. Yaw is induced by mismatching the balance in aerodynamic torques (i.e., by offsetting the cumulative thrust commands between the counter-rotating blade pairs).

iii. Pitch: This is the moving up and down of the quadcopter, similar to nodding. It is controlled the same way as roll, but using the second set of motors. Roll and pitch are determined from where the "front" of the craft is, and in a quadrotor, they are basically interchangeable. The front of the quadcopter must be taken into account in order to be able to ensure consistent control. The pitch is controlled by using the elevator controls on the transmitter.

iv. Roll: This is the tilting left and right of the quadcopter and is controlled by increasing the speed on one motor and lowering on the opposite one, and doing the same on the second set of motors. This can result in a 360-degree sideways roll of the quadcopter. The roll of a quadcopter is controlled using the transmitter aileron controls. 


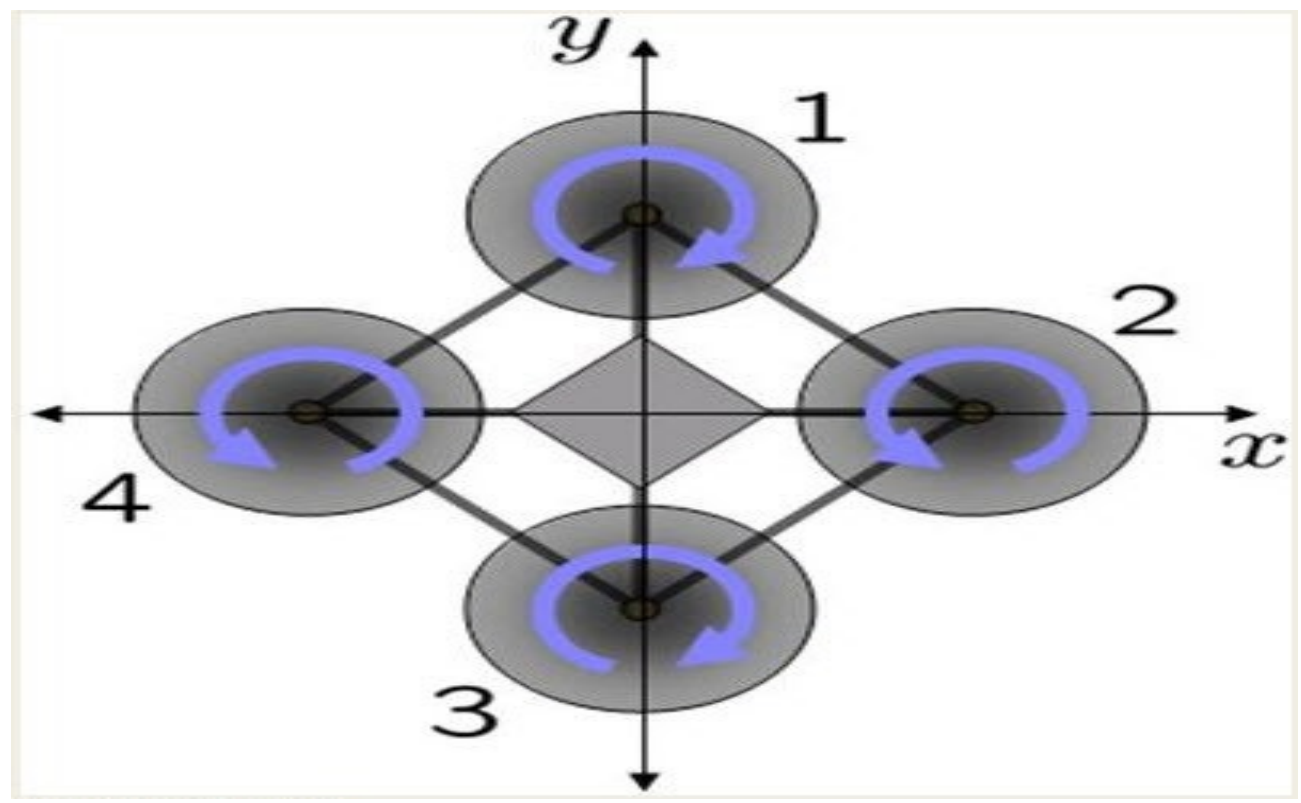

Figure 2: Schematic Diagram of Reaction Torques on Each Motor of a Quadcopter

\section{b. Components of a Quadcopter}

Each quadcopter has a very different hardware component which mostly depends on application in which it is implemented. These components include; the frame, motors, electronic speed controller, flight controller, propellers, radio receiver and transmitter, battery, battery eliminator circuit, power distribution board and optional parts like sensors such as gyroscopes, magnetometers, barometers, accelerometers, sonar, Global Positioning System (GPS) and telemetry modules, Light Emitted Diode (LED) strips, buzzer, battery voltage monitors, cameras and On-Screen Display (OSD) boards. Some of these components are discussed as follows $[1,3,7,9]$ :

i. Frame: The frame of the quadcopter is the main and only supporting structure useable by such a craft. It can be likened to the human skeleton as it carries the weight and bulk of the other component being attached to the quadcopter. A quadcopter frame consists of two to three parts which don't necessarily have to be of the same material: the center plate where the electronics are mounted, four arms mounted to the center plate and four motor brackets connecting the motors to the end of the arms. Although the frame of a quadcopter can be of different forms and can be made of up of different materials, when divided laterally, both side must be of corresponding size and weight in order to ensure a balance of the craft. Materials used to build quadcopter frames can be plastic, wood, carbon fiber, fiber glass, aluminum and even a silicon panel.

ii. Motors: The motor is the unit that rotates the propellers, thus providing thrust, uplift and movement to the quadcopter. Most quadcopters make use of a brushless DC motor. Brushless DC electric motor (BLDC motors, BL motors) also known as Electronically Commutated Motors (ECMs, EC motors) are synchronous motors that are powered by a DC electric source via an integrated inverter/switching power supply, which produces an AC electric signal to drive the motor. Quadcopters generally use higher pole count motors called "out-runners" so that they can avoid using a gearbox. .High pole count motors output a high torque - and this means a gearbox will not be necessary to increase the torque.

iii. Electronic Speed Control: An Electronic Speed Control (ESC) is an electronic circuit with the purpose of varying an electric motor's speed, its direction and possibly also to act as a dynamic brake. ESCs are often used on electrically powered radio controlled models, with the variety most often used for brushless motors essentially providing an electronically generated three-phase electric power low voltage source of energy for the motor. ESCs are an essential component of modern quadcopters and offers high power, high frequency, high resolution 3-phase AC power to the motors in an extremely compact miniature package. These crafts depend entirely on the variable speed of the motors driving the propellers. 
iv. Flight Controller: The flight controller is an on-board microcontroller that acts as a computer and provides flight control capabilities to quadcopters. This allows for autonomous flight, and provides an interface for the connection of sensors to the quadcopters sensors such as cameras, sonar, GPS, Inertial Measurement Units (IMU), which consist of the compass, barometer, gyroscope and accelerometer, telemetry and others. The flight controller is also connected to the ESCs and the receiver and uses the inputs from the sensors to provide a smoother and better flight. If connected to a GPS module, the flight controller can provide autonomous flight of the quadcopter. Flight controllers make use of complex algorithms called PIDs (proportional, integrals and derivatives) to achieve these functions.

Propellers: A propeller is a type of fan that transmits power by converting rotational motion into thrust. In quadcopters, the four propellers provide thrust or uplift, which is the force necessary to lift the craft off the ground. By changing the amount of thrust of each propeller, the quadcopter can be moved forwards, backwards, sideways and rotated. The four propellers on a quadcopter are grouped according to their direction of rotation. Two motors rotate in the opposite directions clockwise to the other two counterclockwise in order to avoid body spinning. By making the propeller pairs spin in each direction, this makes it possible for the craft to stabilize the yaw rotation, which is the rotation around itself. Opposing propellers always move in the same direction. Propellers are specified by their diameter (D) and pitch $(\mathrm{P})$.

vi. Transmitters and Receivers: A transmitter is an electronic device that generates, amplifies and encodes a carrier wave, modulates it with a signal derived from visual, speech and other sources and broadcasts the resulting signal from an antenna. A receiver is an electronic device that accepts this transmitted signal and amplifies or decodes it if necessary and outputs this processed signal as one similar to the original information being transmitted. A quadcopter makes use of two different transmitter/ receiver (Flight Control Transmitter/ Receiver) combinations, one for control of the craft and the other for transmission of video signals. This combination consists of the transmitter which acts as a remote controller, the receiver which receives commands from the transmitter and various sensors that provide output which are sent to the transmitter for display.

vii. $\quad$ Battery: The battery of a quadcopter is the source of power for everything on-board for the craft. Quadcopters make use of a Lithium Polymer ( $\mathrm{LiPo}$ ) battery with a number of cells ranging from two to over ten. Each cell has a voltage of 3.7. The cells in a LiPo battery are connected in series, and the total power of the battery is the product of the cell voltage and the number of cells present. That is, a threecell battery denoted $3 \mathrm{~S}$ has a total output voltage of 11.1 . The size, rating and output of a battery can vary widely, depending on the maximum current drawn, motors, size and weight of the craft and the amount of flight time desired. The flight time provided by a battery depends on the type of motors, propellers, on-board electronics, flight style of the pilot, flying conditions and the weight of the craft. While, a larger capacity battery can provide more flight time, larger capacity batteries are heavier thus causing a reduction in the flight time due to added weight and increased torque.

Battery Eliminator Circuit: In most cases, only the motors are connected directly to the battery as the battery voltage is too high for most of on-board electronics. This problem is eliminated by the use of a Battery Eliminator Circuit (BEC). The BEC is an electronic circuit designed to deliver electrical power to other circuitry without the need for multiple batteries. BECs in their simplest form use a linear fixed voltage regulator and regulate the voltage from the battery to the $5 \mathrm{~V}$ used by on-board electronics such as the receiver and flight controller, and also the $12 \mathrm{~V}$ used by the FPV camera.

Power Distribution Board: The Power Distribution Board (PDB) is used to distribute power on a quadcopter and to avoid multiple ESC to battery connections by providing a neat and clean platform for the connection of all devices to the battery. The PDB is a basic circuit board that connects all the ground connectors to one another and all the positive connectors together.

Camera: A camera is an optical instrument for recording or capturing images, which may be stored locally, transmitted to another location, or both. The images may be individual still photographs or sequences of images constituting videos or movies. The camera is a remote sensing device as it senses subjects without physical contact. There are two types of cameras used on quadcopters; First Person View (FPV) cameras for piloting and professional cameras used for aerial photography.

On-Screen Display: An On-Screen Display (OSD) is an image superimposed on a screen picture, commonly used by modern television sets, VCRs, and DVD players to display information such as volume, channel, and time. In quadcopter, OSD is used to display additional data from sensors on the live FPV feed being transmitted to the user. This data can include location coordinates, altitude, 
atmospheric temperature and pressure, wind speed and direction, flight time, on-board battery voltage, orientation and compass direction, distance from pilot, RPM and the speed of the craft.

xii. On-Board Sensors: On-board sensors are electronic measurement units that measure different values ranging from temperature, atmospheric pressure, wind speed, direction and altitude.

\section{c. Applications of Quadcopters}

Quadcopters exist in different sizes and configurations and are used in different aspects and for diverse uses ranging from aerial photography, surveillance, inspection, reconnaissance and for delivery. The most important applications of quadcopters are discussed as follows [6, 13]:

i. Research and Test Platforms: Quadcopters are used globally by industrial researchers to evaluate and test new ideas in different fields such as flight control theory, navigation, real time systems and robotics. Swarms of quadcopters can hover and fly in formations and perform complex flying routines. Advantages of using quadcopters as test platforms are numerous as they are relatively cheap, available in a variety of sizes and their simple mechanical design means that they can be built and maintained by amateurs.

ii. Military and Law Enforcement: Quadcopters are used for surveillance and reconnaissance by the military and other law enforcement agencies as well as search and rescue missions in urban environments.

iii. Commercial use: The bulk of quadcopter applications can be ascribed to commercial uses. Commercial uses of quadcopters include aerial photography and inspection of property such as power transmission lines and pipelines. Quadcopters are more suited to these uses than other UAVs due to their autonomous nature, maneuverability and lower construction costs.

\section{DESIGN OF AN IMPROVED QUADCOPTER}

The design of an improved quadcopter was carried out with a particular set of specifications. These specifications, which are stated in Table 1 include the size of the frame (Wheel base which is the length of the base of a motor to the base of the motor opposite), total thrust needed for lift, total weight of the quadcopter (which is dependent on the thrust), flight time, maximum height of climb, type of receiver technology, type of battery, size and type of propellers, type of camera, transmitter mode of operation and with a cost of no more than 250 USD. With these specifications parameters, components were selected and acquired

Table 1: Design specifications and parameters.

\begin{tabular}{ll}
\hline Specifications & Parameters \\
\hline Size of Frame (Wheelbase) & $500 \mathrm{~mm}$ \\
Total Thrust & $2500-3100 \mathrm{~g}$ \\
Total Weight & $1250 \mathrm{~g}$ \\
Flight Time & $10-20$ minutes \\
Maximum Height & $>400 \mathrm{~m}$ \\
Receiver Type & PWM \\
Battery Type & $3 \mathrm{~S}, 2500-3500 \mathrm{mAh}, 25 \mathrm{C}$ \\
Propeller Type and Size & Plastic props, 10x4.5in \\
Camera Type & HD Video with On-board Storage \\
Transmitter Mode & Mode 2 \\
Maximum Current Draw & $60 \mathrm{~A}$ \\
\hline
\end{tabular}

a.

\section{Components Used in the design of an improved Quadcopter}

Some of the components used in the design of an improved quadcopter are:

i. Quadcopter Frame: The frame used in this study is the S500 quadcopter frame with the integrated center plate. This frame has an integrated power distribution board and camera gimbal. The PDB center plate is made of fiberglass while the arms and landing gears are plastic. The wheel base of the S500 frame is $500 \mathrm{~mm}$ and it has a total weight of $404 \mathrm{~g}$. The total height is $190 \mathrm{~mm}$ with a leg spacing of $234 \mathrm{~mm}$. The S500 frame is a scaled-up version of the DJI F450 Flame wheel Quadcopter. The S500 frame has two variants; one with an integrated PDB fiberglass center plate and the other with a carbon fiber center plate. Figure 3 shows the parts of the frame before assembly. 


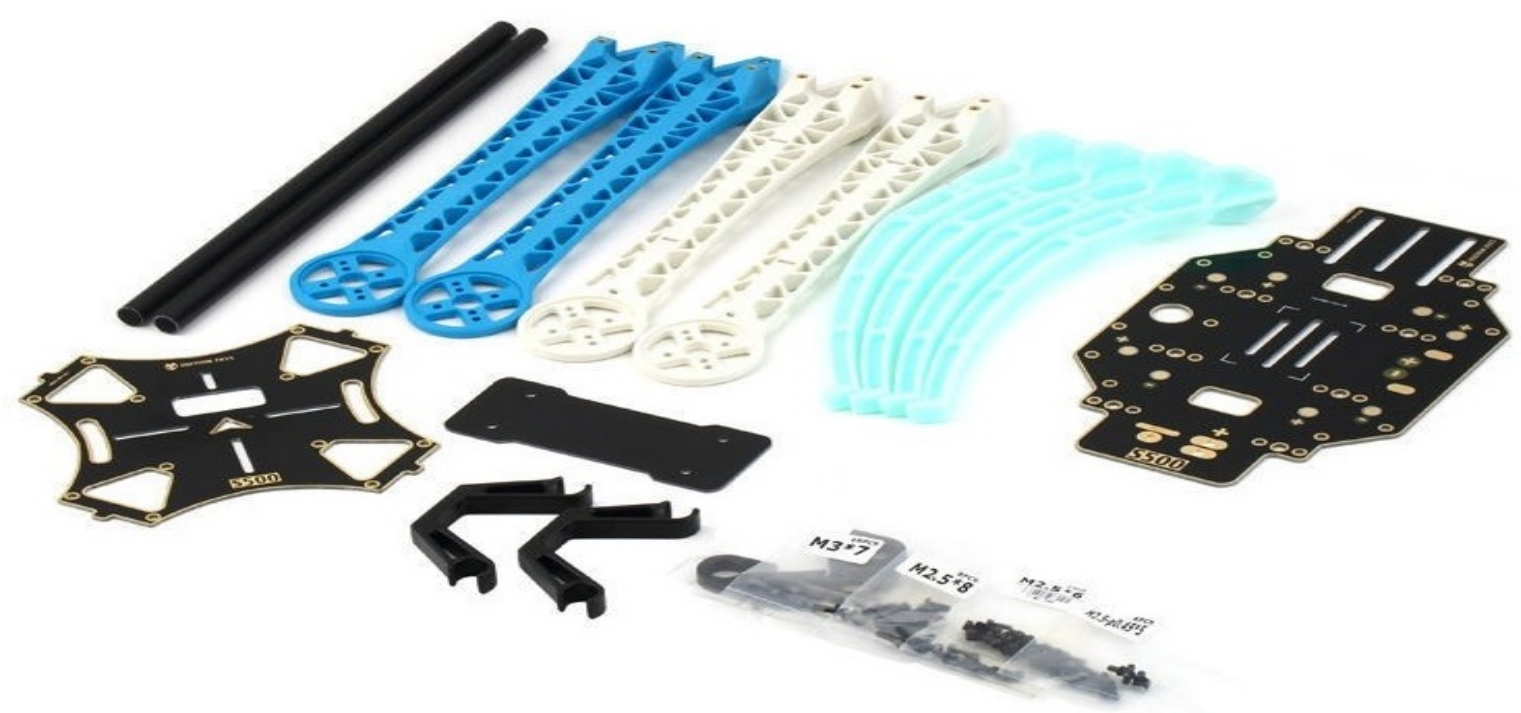

Figure 3: Parts of the S500 Frame before Assembly

ii. Motors: The type of motor used for this study is Emax MT2213. Emax MT2213 is a $935 \mathrm{kV}$ motor capable of producing up to $850 \mathrm{~g}$ of thrust. It comes in both $\mathrm{CCW}$ and $\mathrm{CW}$ modes. The $\mathrm{CW}$ motors have a red screw cap while the CCW motors have a black screw cap for identification. The MT2213 has its prop adapter built into the motor and is made with silicone steel and has a weight of $55 \mathrm{~g}$. When used with a $3 \mathrm{~S}$ battery, 1045 size propellers are recommended. Figure 4 shows the Emax MT2213 motors. The Revolution Per Minute (RPM) of the motor at $11.1 \mathrm{~V}$ is given as:

$$
R P M=k V \times V
$$

\section{$935 \times 11.1 \approx 10,379 R P M$}

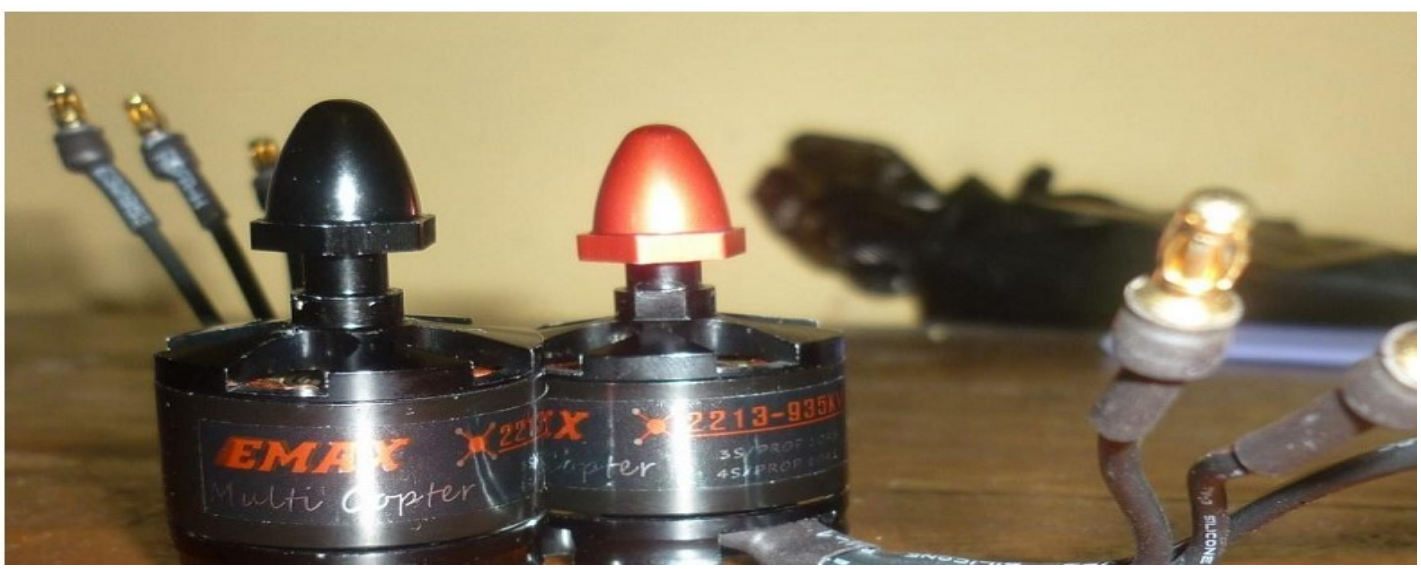

Figure 4: Emax Mt2213 CCW and CW BLDC Motors.

iii. Electronic Speed Controller: The SimonK 30A ESC made by Readytosky shown in Figure 5 is an ESC with an in-built battery eliminator circuit and on-board flashable SimonK firmware. The ESC has a continuous current of $30 \mathrm{~A}$ and a burst current of $35 \mathrm{~A}$. The on-board BEC is a linear mode BEC with an output voltage and current of $5 \mathrm{~V}$ and $3 \mathrm{~A}$ respectively. It makes use of a crystal oscillator and has a 100 $\%$ efficiency N-FET design. It also has a $490 \mathrm{~Hz}$ response rate as well as a motor frequency of $16 \mathrm{kHz}$. It has a total weight of $25 \mathrm{~g}$. 


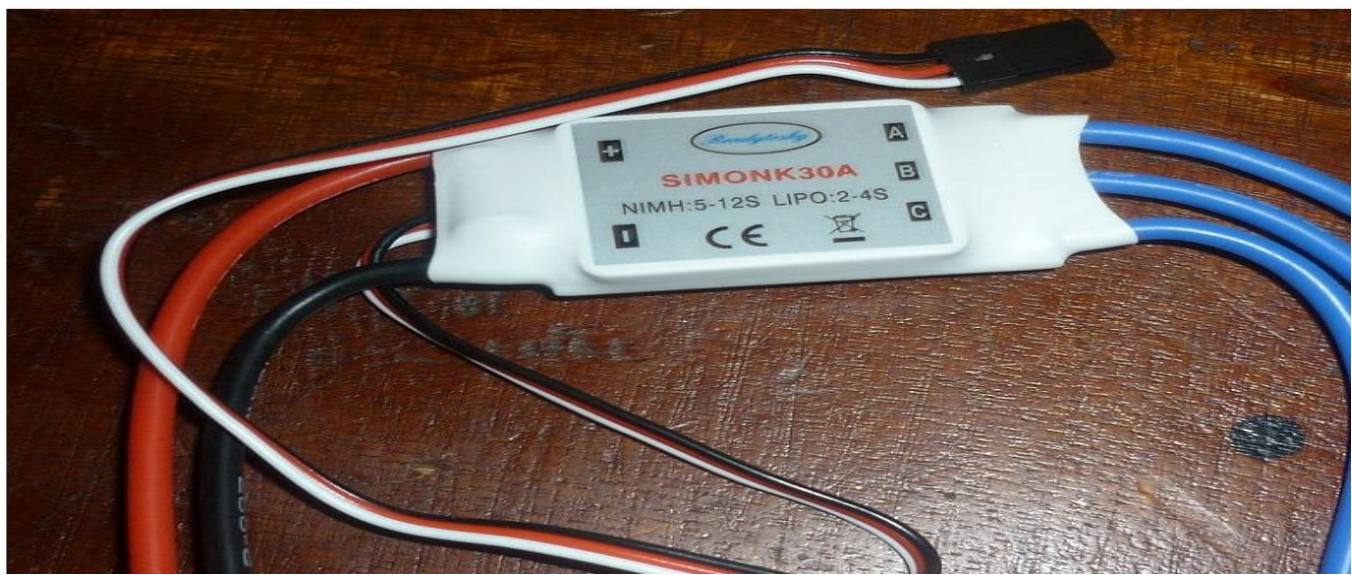

Figure 5: SimonK 30A Electronic Speed Controller.

iv. Flight Controller: The flight controller variant used in this research paper is the 10D0F board which has a 16Mbit on-board memory, a MEMS BMP280 barometer, magnetometer and MPU6500 gyroscope and accelerometer. The board is powered by a $5 \mathrm{~V}$ dc power from the BEC. It can support FrSky telemetry, GPS, sonar, battery monitoring, I2C bus, CPPM or PWM, SBUS, softserial and spectrum satellite which also has a micro USB port which together with its case weighs $13.3 \mathrm{~g}$. The 10D0F Naze 32 is shown in Figure 6.

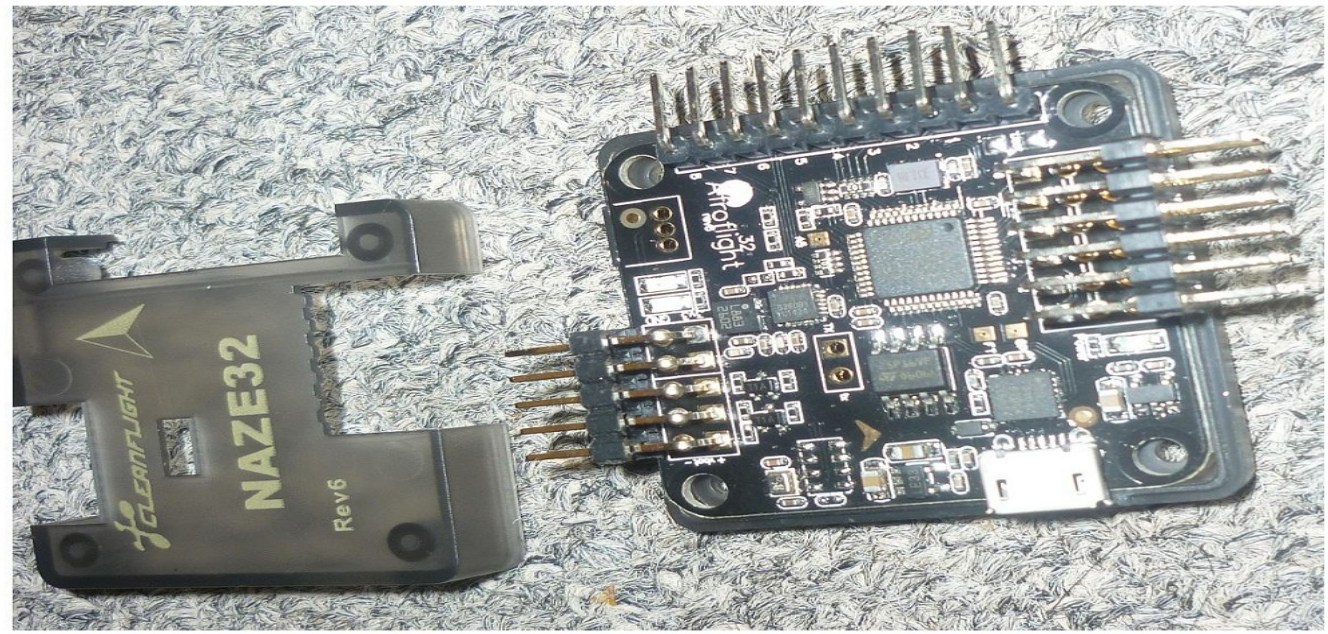

Figure 6: Naze32 REV6 10D0F Flight Controller.

v. Propellers: The propellers used in this study are the LJI 1045 propellers. These propellers are made of Acrylonitrile Butadiene Styrene (ABC) plastic. They have a diameter of 10inches and a pitch (lift per complete revolution) of 4.5 inches. These weigh an average of $9.23 \mathrm{~g}$. The $1045 \mathrm{CW}$ and $\mathrm{CCW}$ variants are shown in Figure 7. 


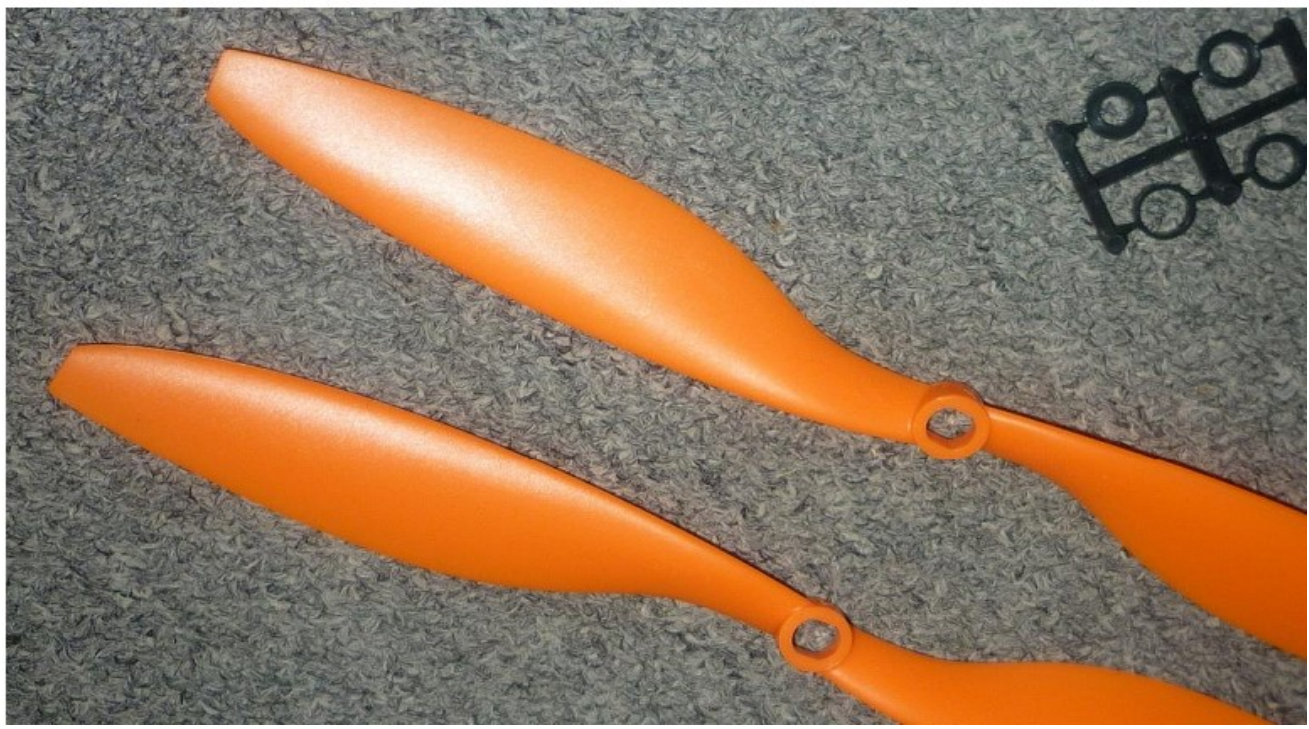

Figure 7: LJI 1045 Propellers.

vi. Transmitter and Receiver: The transmitter and receiver combination used in this study is the FlySky FSi6 transmitter and the FS-iA6 receiver. The FS-i6 is a $2.4 \mathrm{GHz}$ digital proportional radio system with a 2A automatic frequency hopping digital system (AFHDS2A). This ensures a long range jamming free signal range. The FS-i6 has two $26 \mathrm{~mm}$ multidirectional antennas (upgradable) which allows it to attain a range of $500-800 \mathrm{~m}$ out of the box. Its radio frequency range is $2.4056-2.475 \mathrm{GHz}$, with a channel bandwidth of $500 \mathrm{kHz}$. It has a RF power of less than $20 \mathrm{dBm}$, sensitivity of $-105 \mathrm{dBm}$ and uses a Gaussian frequency shift keying (GFSK) modulation method. The Fs-i6 is a 6-channel transmitter with channels 1-4 used for the control sticks and channels 5-6 used for auxiliary controls. It has a backlit LCD display screen for on-board settings and is powered by 4 AA batteries and a trainer cable/ firmware upgrade port. Figure 8 shows the Fs- 16.

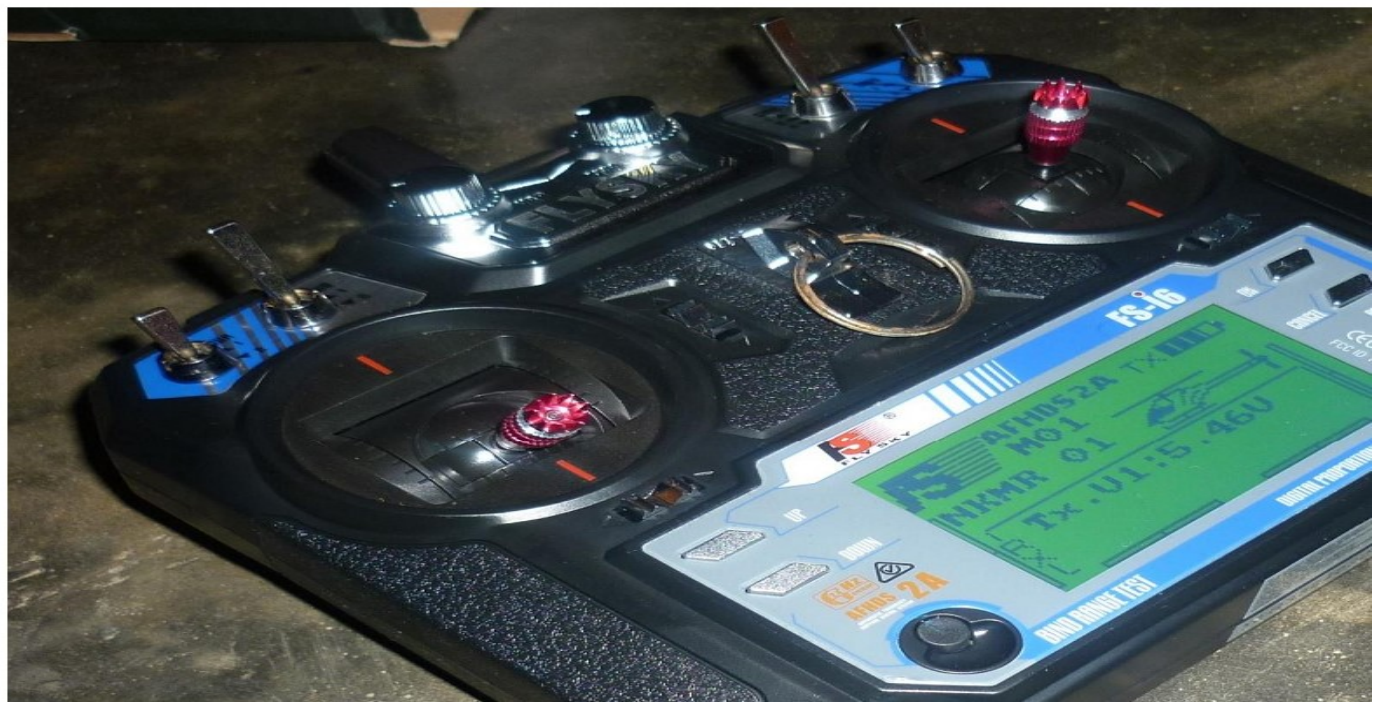

Figure 8: FlySky FS-i6 Transmitter.

vii. $\quad$ Battery: The Tiger-Power Atomic-Platinum battery used in this study is a 3S $3500 \mathrm{mAh}$ LiPo battery with a discharge rate of $30 \mathrm{C}$ and a voltage of $11.1 \mathrm{~V}$. The battery comes with a XT60 female port for power connection with a weigh of $281.4 \mathrm{~g}$. This is shown in Figure 9. 


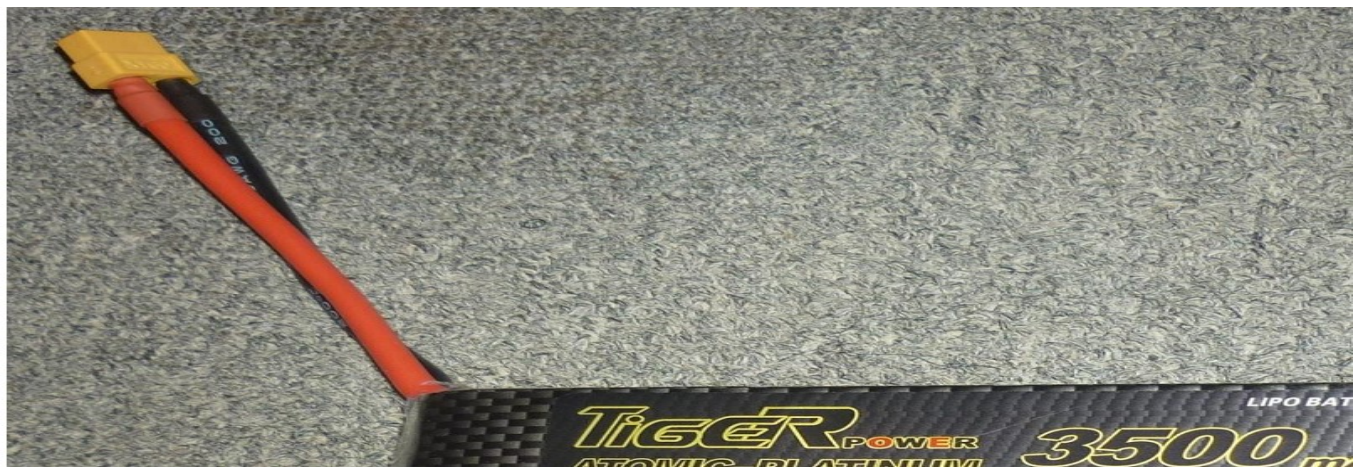

Figure 9: Tiger-Power 3500mAh LiPo Battery.

viii. Camera: The camera used is the SQ8 Mini DV 12MP full HD camera shown in Figure 10. The SQ8, originally designed as a spy camera was chosen due to its HD and night vision capabilities and also its small size and onboard picture processing abilities. This enables the camera to work without a direct-tocontroller link as images are stored on an onboard memory card.

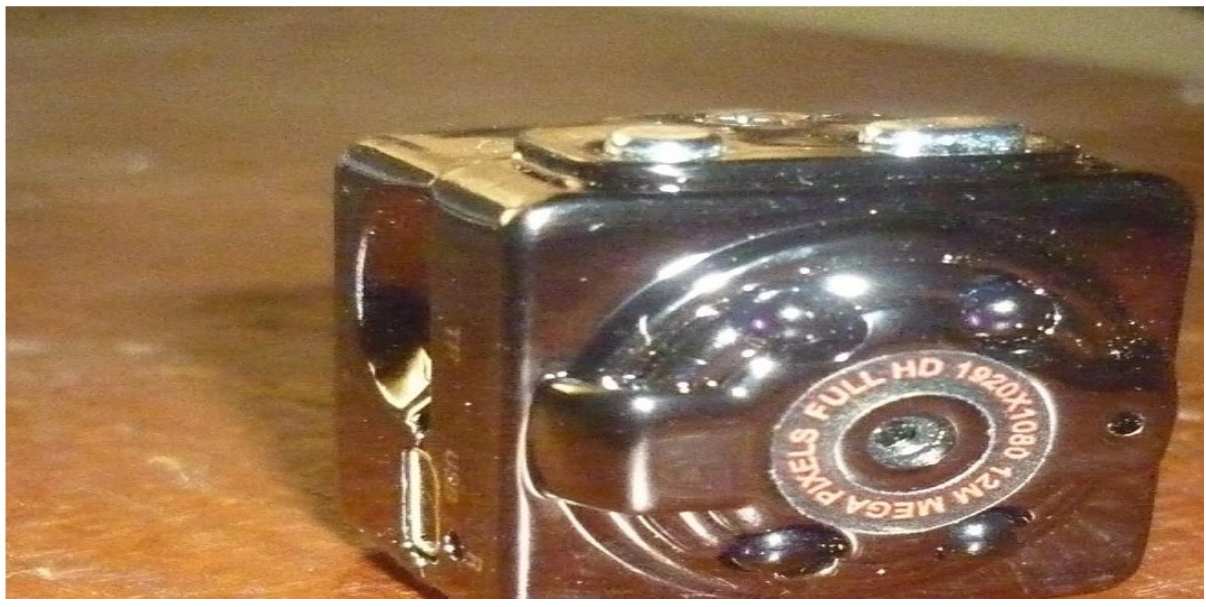

Figure 10: SQ8 Mini DV Camera.

\section{b. Simulation Software}

The software used for configuring the flight controller is the Cleanflight Configurator version 2.1.0. The Cleanflight Configurator is an offshoot of the Betaflight Configurator which is a Chrome desktop browser application. The firmware run on the flight controller is the Cleanflight Hex v2.1.0, 2017.

\section{ELECTRICAL COMPONENTS OF THE IMPROVED QUADCOPTER}

The electrical components of the quadcopter include the motors, ESCs, the flight controller, the receiver, buzzer and the battery. Since the ESCs have built-in BECs, the 5Vdc power input for the flight controller and receiver was provided by the ESCs. Each ESCs signal cable with the positive and ground wires are connected to servo ports 1-4 according to the corresponding motors. Figure 11 shows the assembly pictures of the frame while Figure 12 shows the electrical connections of the drone.

The battery voltage monitoring port is the only part of the flight controller that can be connected directly to the battery. This port can handle voltage within the range of 5-26 V and is used for in-board battery voltage monitoring with the use of a buzzer for low battery alerts. 

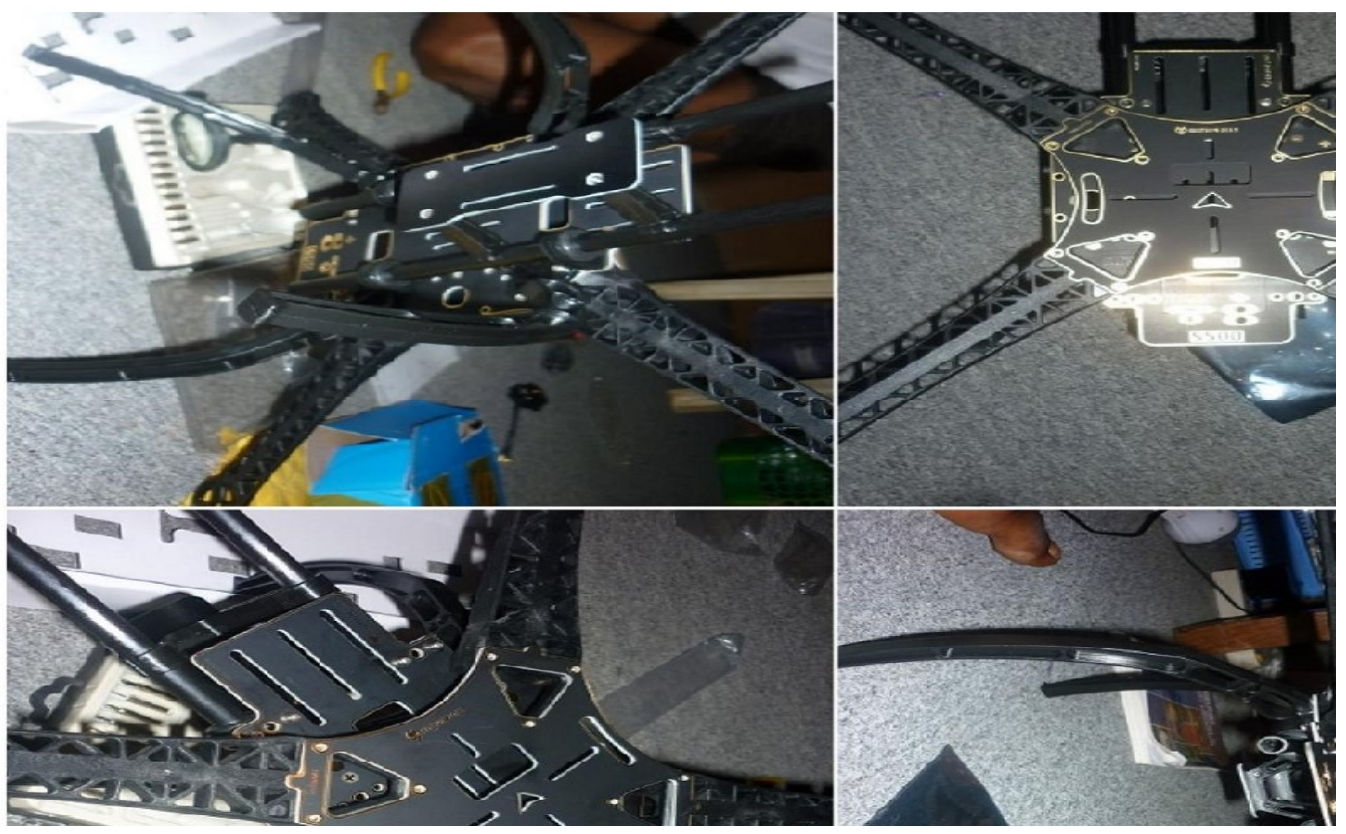

Figure 11: Images of the Frame after Assembly

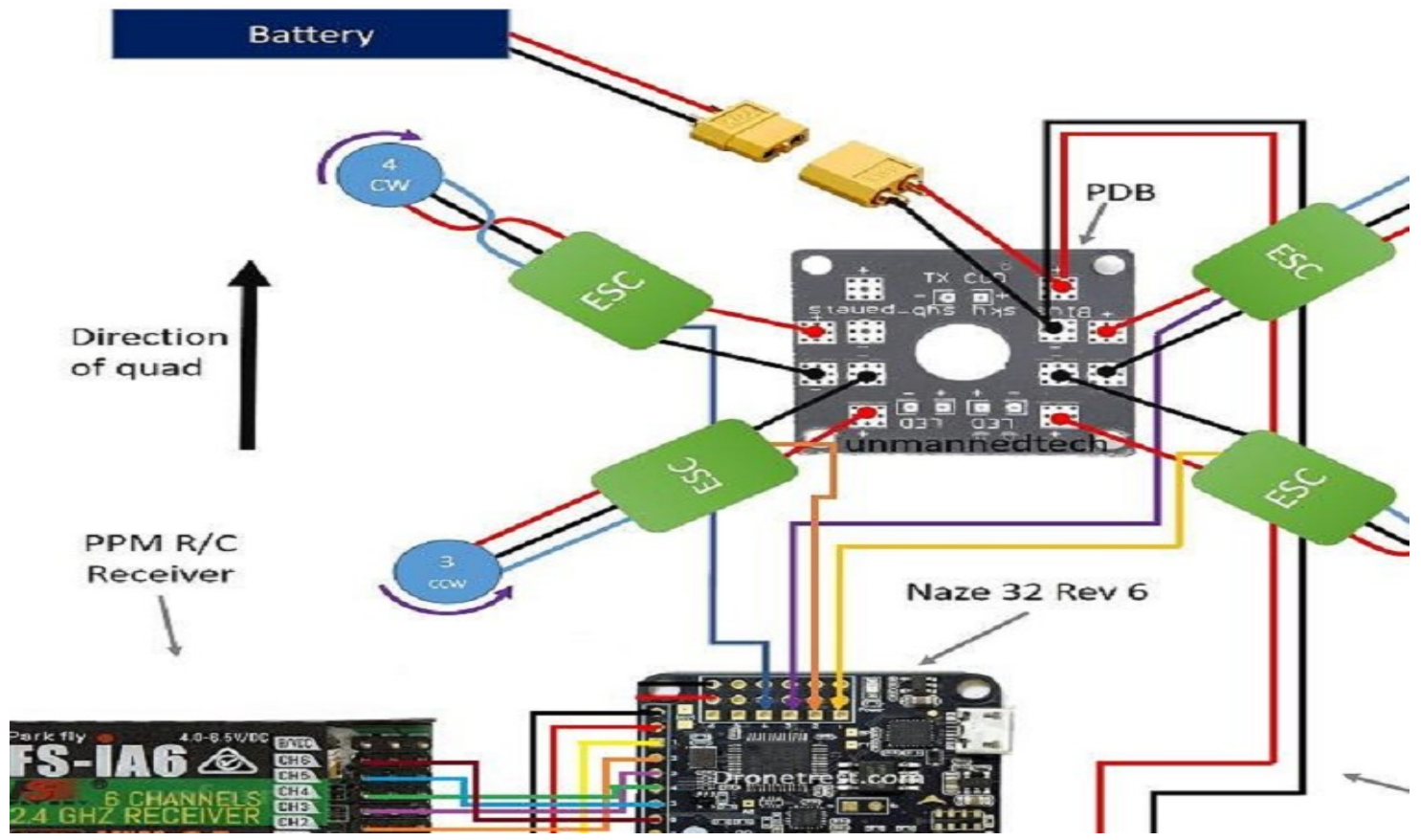

Figure 12: Connection of Electrical Components 


\section{a. $\quad$ Flight Controller Configuration}

All configurations of the flight controller were done with the Cleanflight Configurator desktop software version 2.1.0, while the firmware run on the controller is the Cleanflight 2.1.0 Hex (the highest and last firmware release for the Naze32 board). These were the steps taken in configuring the flight controller:

i. Installation of the CP210x drivers on the system used for configuration.

ii. Flashing the firmware to be run on the PC. This was done by using the Firmware Flasher tab. No reboot sequence full chip erase and manual baud rate options should be left unchecked for a first time firmware installation..

iii. After the firmware is installed, the board is connected by clicking the connect button on the top right corner of the configuration welcome page.

iv. On the setup page, the on-board accelerometer is calibrated by placing the quadcopter on a flat level surface. The $\mathrm{z}$-axis heading was also reset if the virtual drone image is not facing forward.

v. On the configuration page, Quad X (frame configuration) was selected. ESC/ Motor protocol was set as PWM. Motor Stop and Disarm Motors were selected in order to stop the motors from spinning immediately the quadcopter is armed. The minimum throttle value was set as 1150 , maximum throttle was set at 2000 and minimum throttle command was set at 1000 .

vi. $\quad$ Since the on-board gyroscope is a MPU6500 MEM sensor, 32KHz sampling mode (sensor refresh rate) was selected. The Gyro update frequency and PID loop frequency were set at $2 \mathrm{kHz}$ and $0.5 \mathrm{kHz}$ respectively.

vii. On the Power and Battery page, the minimum and maximum cell voltages were set at $3.7 \mathrm{~V}$ and $4.3 \mathrm{~V}$ respectively. The warning voltage was set at $3.8 \mathrm{~V}$. On-board ADC was selected as the voltage meter source.

viii. On the Failsafe page, the Enable failsafe 2 tab was checked and failsafe procedure was set as land. Landing throttle value was set at 1100 . All other settings were left at their default values.

ix. On PID Tuning page, the default PID values were left at the default values. Changes to the PID values were done after flight in order to correct drifting.

x. On the Receiver page, AETR1234 was selected as the channel map. Stick min, stick center and stick max values were set as 1050,1500 and 2000 respectively.

xi. On the Modes page, Aux channel 2 was set to arm and disarm. Aux channel 1 was set to three modes: Horizon, Angle and Acro modes.

xii. On the Motors page, motor control was checked and the master slider was raised to the maximum. The battery was plugged into the drone and the slider was brought back down. This process was used to calibrate the ESCs.

xiii. On the Blackbox page, the on-board flash memory was used to record in-flight information. Thgbfis information was saved and viewed in order to troubleshoot in-flight problems.

xiv. The Command Line Interface (CLI) page was used to make command line based changes to the configurations. Figure 13 shows the screen display of Cleanflight Configurator used. 


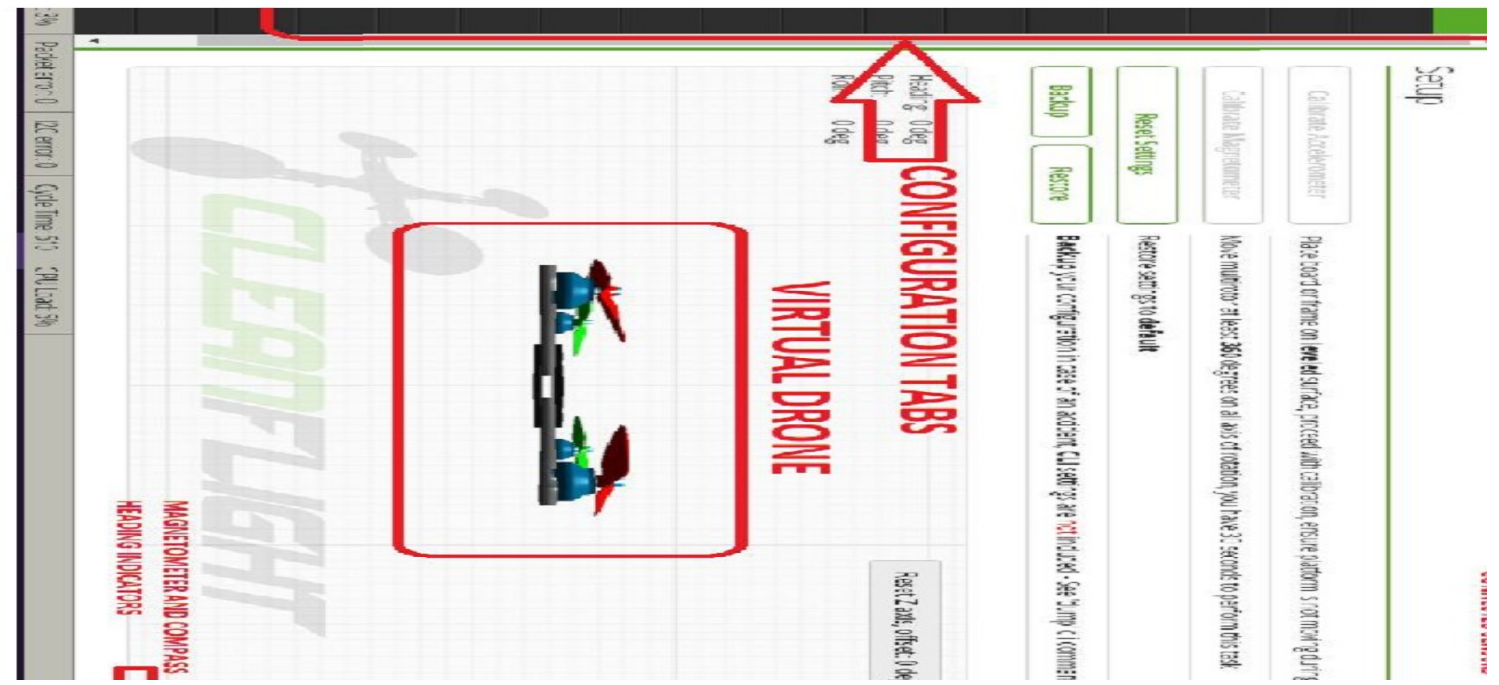

Figure 13: Cleanflight Configurator

\section{b. Transmitter Configuration}

Transmitter configurations include channel trimming, start and end point calibration, receiver binding, expo/rate settings, switches assigning, model naming, selection and student trainer modes. The receiver is factory-bound to the transmitter and other configurations are done at the pilot's consideration as the flight was achieved at the default settings.

\section{c. Camera Configuration}

The camera was mounted on a custom-made hinged hook attached to the gimbal base plate provided with the frame. The hinge is a laptop computer lid chosen because of its swivel angles and its rigidity and ability to resist shock and vibrations. The hook was improvised from the desktop base hook provided with the camera. The mount is shown in Figure 14. The SQ8 camera, due to its small size has only two buttons on it, the ON/OFF button and MODE select button.

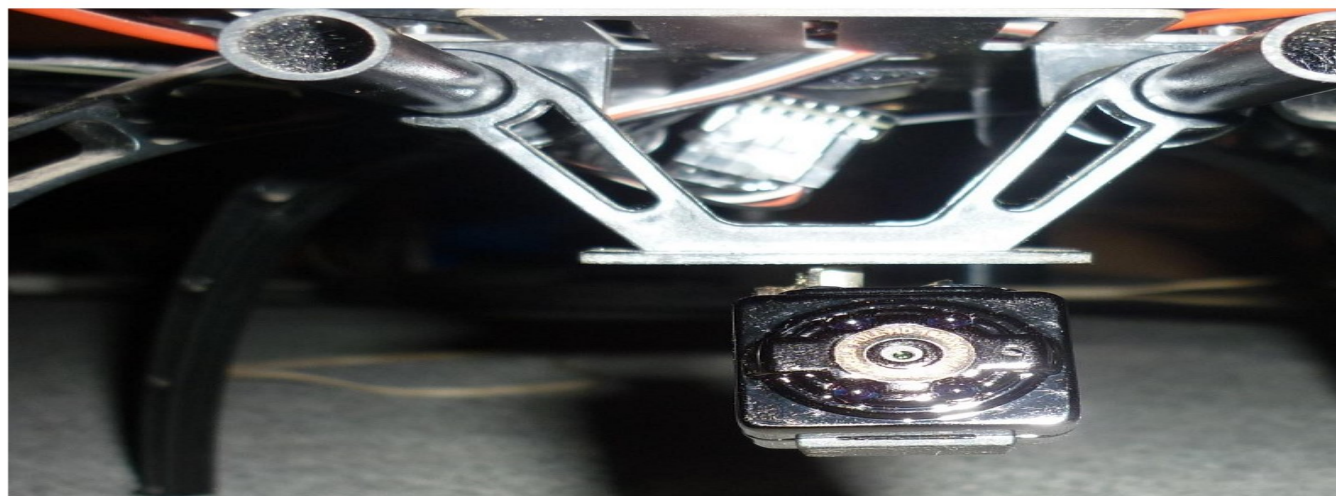

Figure 14: Camera Mount

\section{d. Battery Mounting}

The battery is mounted on the top plate quadcopter with the use of a Styrofoam padded Velcro battery strap. This foam padding is necessary to provide enough grip so as to prevent the battery from slipping during flight.

\section{e. Total Weight}

The total weight of the quadcopter after assembly is approximately $1.2 \mathrm{~kg}$. This is inclusive of every component- electrical and mechanical componenst with the exception of the transmitter. Figure 15 shows the complete assembly of the quadcopter. 


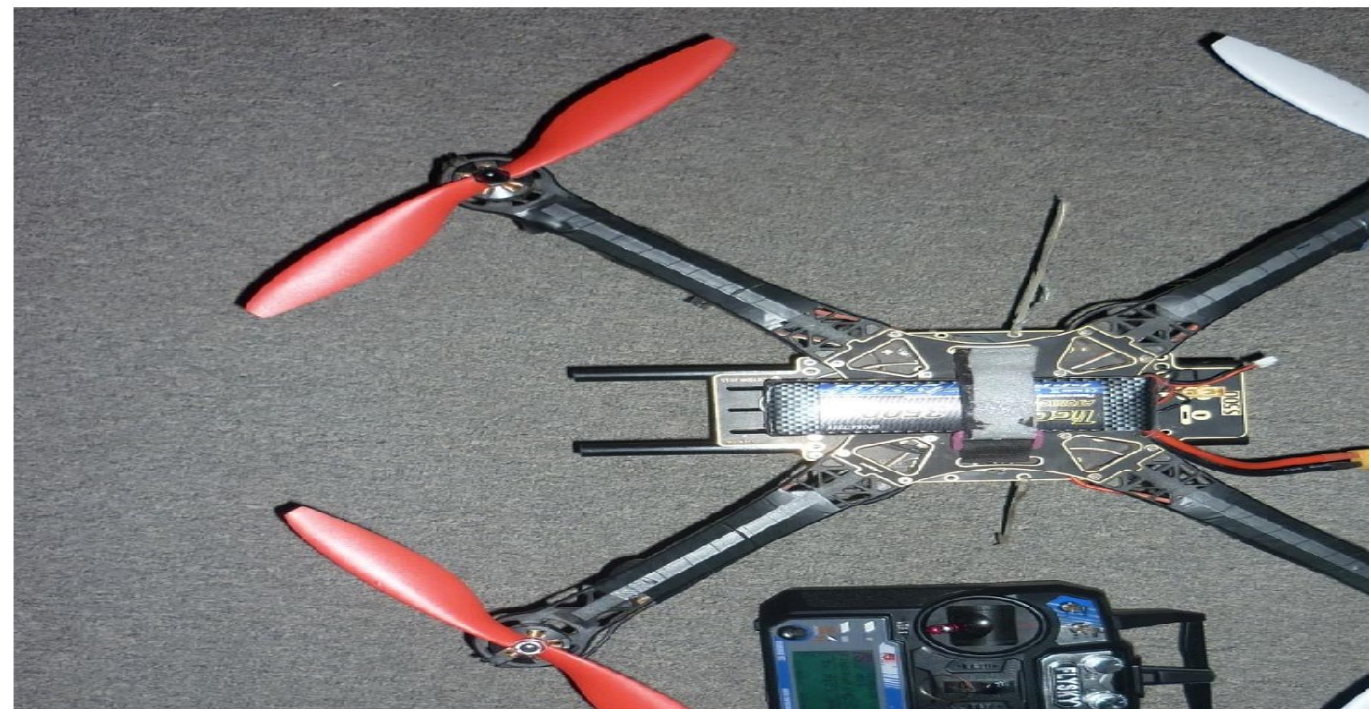

Figure 15: Image of Quadcopter After Assembly

\section{CONCLUSION}

An improved industry-grade quadcopter which can be used for diverse purposes such as surveillance, aerial photography, payload- deliver and aerodynamic has been designed and implemented. The basic principles of quadcopter design as well as current applications have been presented. The study has shown that Small Unmanned Aerial Vehicles (SUAVs) are useful across a broad range of applications. However, operation and piloting of this drone is only recommended for pilots who have passed flying on a simulator. This is because an inexperienced pilot can cause damage to onlookers present and even to the drone itself.

In addition, with the emergence and popularity of electric powered vehicles due to the damaging effect of fossil fuel combustion on the environment, it is advisable that design engineers should take a more proactive step in the development of high efficiency electronic components and circuits.

\section{REFERENCES}

[1] Axel, R. (2017): "Position and trajectory control of a quadcopter using PID and LQ controllers", Master of Science Thesis in Electrical Engineering, Division of Automatic Control Department of Electrical Engineering Linköping University, Sweden, Pp: 1-81.

[2] Dronetest, P. (2011): "Power distribution boards-how to choose the right one", http:/www.dronetrest.com/t/power-distribution-boards-how-to-choose-the-right-one/1259, Pp: 1-10.

[3] Dryden, J. and Barbaccia. R. (2014): “Quadcopter design project”, ME 445 Final Report, Pp: 1-54.

[4] Finn, R. L. and Wright, D. (2012): "Unmanned aircraft systems: surveillance, ethics and privacy in civil applications", Computer Law and Security Review, Vol. 28(2), Pp: 184-194.

[5] Ingrid, K. (2016): "Black-box modeling and attitude control of a quadcopter", Master of Science Thesis in Electrical Engineering Department of Electrical Engineering, Linköping University, Pp: 1-68.

[6] Jeremia, S., Kuantama, E. and Pangaribuan, J. (2012): "Design and construction of remote-controlled quad-copter based on STC12C5624AD”, International Conference on System Engineering and Technology September 11-12, 2012, Bandung, Indonesia, IEEE Transaction, Pp: 1-7.

[7] Jessie, D., Frederick, K. and Leonardy, S. (2011): “Color recognition robot”, In Proc. ICCCD, 2011, Pp.85-89.

[8] Kandaswamy, G. (2011): "Drone based sensor platforms", TCS Research and Innovation, Pp: 1-44.

[9] Oscar L. (2009): "Choose flight controller for quadcopter", https://oscarliang.com/best-flight-controllerquad-hex-copter/, Pp:1-8.

[10] Oscar L. (2014): "Build a quadcopter from scratch-hardware overview" http://blog.oscarliang.net/build-a-quadcopter-beginners-tutorial-1, Pp: 1-10. 
[11] Oscar, L. (2016): "Types of multitrotor" https://oscarliang.com/types-of-multicopter/, Pp; 1-20.

[12] Ostojić, G., Stankovski, S., Tejić, B., Đukić, N. and Tegeltija, S. (2015): "Design, control and application of quadcopter", International Journal of Industrial Engineering and Management (IJIEM), Vol. 6(1), Pp. 43-48.

[13] Quadcopter (2014): “The Unmanned Aerial Vehicle” http://aermech.com/quadcopter-unmanned-aerialvehicle/ Pp: 1-20. 